\title{
Validación Transcultural del Cuestionario de Habilidades Sociales para Universitarios en Estudiantes Chilenos y Argentinos (CHS-U)
}

\author{
Transcultural Validation of Social Skills Questionnaire for College Students (SSQ-U) \\ from Chile and Argentina
}

\author{
Valeria E. Morán ${ }^{1}$, Felipe E. García ${ }^{2}$ y Pedro Hormazabal ${ }^{3}$
}

\section{Resumen}

El objetivo del presente estudio es realizar una validación transcultural del Cuestionario de Habilidades Sociales para Universitarios de Morán et al. (2015) en estudiantes chilenos y argentinos. Con este fin, se evaluó a 1025 estudiantes universitarios entre 20 y 25 años, de los cuales $61.2 \%$ fueron mujeres y $38.8 \%$ hombres. De ellos, 771 son argentinos y 254 chilenos. Se realizó un análisis factorial confirmatorio que encontró las cinco dimensiones del instrumento original, y una prueba de invarianza factorial comparando a estudiantes argentinos y chilenos, obteniendo evidencia de invarianza configural y métrica entre los dos grupos. El instrumento muestra una adecuada consistencia interna tanto para el instrumento completo como cada una de sus dimensiones. Estos resultados permiten contar con un instrumento válido y confiable para la evaluación de las habilidades sociales en estudiantes de ambos países.

Palabras clave: habilidades sociales, análisis factorial confirmatorio, invarianza factorial, estudiantes universitarios, validación transcultural

\begin{abstract}
The aim of this study is to conduct a cross-cultural validation of the Social Skills Questionnaire for College Students by Morán et al. (2015) in Chilean and Argentinean students. 1025 university students, 771 from Argentina and 254 from Chile altogether between 20 and 25 years old, $61.2 \%$ women and $38.8 \%$ men, were evaluated. We carried out a confirmatory factor analysis that found the five dimensions of the original instrument, and a factorial invariance test comparing Argentine and Chilean students, obtaining evidence of configural and metric invariance between the two groups. The instrument shows an adequate internal consistency both for the complete instrument and for each of its dimensions. These results allow us to have a valid and reliable instrument for the evaluation of the social skills in students from both countries.
\end{abstract}

Keywords: social skills, confirmatory factor analysis, factorial invariance, university students, crosscultural validation

\footnotetext{
1 Dra. en Psicología. Instituto de Investigaciones Psicológicas- IIPsi [CONICET- UNC]. Facultad de Psicología, Universidad Nacional de Córdoba, Argentina. Enfermera Gordillo s/n. Ciudad Universitaria, Córdoba, Argentina. Correo: moranvaleria@gmail.com

${ }^{2}$ Doctor en Psicología, Universidad de Concepción, Chile. Académico Facultad de Ciencias Sociales, Universidad Santo Tomás, Chile. Arturo Prat 885, Concepción, Chile. Correo: fgarcia@ santotomas.cl

3 Psicólogo, Universidad de Las Américas, Chile. Chacabuco 539, Concepción, Chile. Correo: pdrx.hormazabaln@gmail.com
} 


\section{Introducción}

En la actualidad el estudio de las habilidades sociales (HHSS) se ha convertido en un área de innegable importancia y gran productividad. Son múltiples los estudios que aportan evidencia acerca de su relevancia no solo en el área clínica sino también en el área educacional, laboral y jurídica, entre otras (Bunford, Evans, Becker, \& Lanberg, 2015; Deming, 2015; Gil Rodríguez, Cantero Sánchez, \& Antino, 2013; Gutiérrez Carmona, \& Expósito López, 2015; Olivares, Olivares-Olivares, \& Maciá, 2014; Sofronoff, 2016). Desde los primeros estudios, este campo de estudio se ha visto enriquecido por los aportes de múltiples investigadores de todo el mundo, quienes han aportado diferentes visiones $\mathrm{y}$ conceptos, intervenciones e instrumentos de evaluación.

Desde un enfoque cognitivo comportamental se define a las HHSS como el repertorio de conductas que permiten al individuo relacionarse eficazmente con otras personas. Estas conductas permiten manifestar actitudes, deseos, opiniones o derechos de un modo adecuado a una situación, respetando la expresión de esas conductas en los demás (Caballo, 2007). Estas respuestas conductuales que resuelven habilidosamente una interacción interpersonal son precedidas por una serie de sucesos cognitivos y ambientales que interactúan entre sí y son afectados a su vez por las respuestas conductuales emitidas (Olaz, 2012). Gresham (2011) define las HHSS como "comportamientos aprendidos y socialmente aceptables que permiten al individuo interactuar efectivamente con otros y evitar comportamientos no aceptables que resulten en interacciones sociales negativas" (pp. 21). Por su lado, Kelly (2002), añade que estas conductas buscan obtener o mantener refuerzos del ambiente para el establecimiento de relaciones con los demás.

Vizcarro (1994) afirma que el estudio de las HHSS tiene implicaciones en diversos ámbitos, tales como laboral, educacional, clínico y psicología del desarrollo, entre otros. Desde sus inicios en Inglaterra y EEUU, este campo se ha extendido a Latinoamérica, donde no solo se han llevado a cabo estudios empíricos (Da Costa \& Del Prette, 2012; Hidalgo \& Abarca, 2000; Del Prette, Del Prette, \& Barreto, 1999; Herrera,
Freytes, López, \& Olaz, 2012; Pedraza, Socarrás, Fragozo, \& Vergara, 2014; Reche \& Lucena, 2016) sino que también se cuenta con importantes desarrollos teóricos (A. Del Prette \& Z.A.P. Del Prette, 2003, 2008, 2014; Z.A.P Del Prette \& A. Del Prette, 2002, 2005, 2011; Olaz \& Morán, 2014).

Coincidiendo con Tornimbeni, Pérez y Olaz (2008), en Psicología la mayor producción científica en un área específica está muchas veces relacionada a la posibilidad de contar con instrumentos de evaluación para los constructos estudiados, tal como evidenciaron Prytz Nilsson, Suarez y Olaz (2014) en su estudio bibliometrico sobre HHSS. En este trabajo, los autores mostraron que el crecimiento de la producción a nivel teórico y de investigación empírica sobre esta temática en Latinoamérica ha posibilitado el desarrollo paralelo de instrumentos de evaluación.

En el caso de Chile, los estudios empíricos específicos sobre las HHSS que han sido publicados son escasos. Algunos de ellos se han centrado en proponer instrumentos para su medición en estudiantes universitarios (Abarca \& Hidalgo, 1989; Miranda, Riquelme, Cifuentes, \& Riquelme, 2014); otro estudio se centró en establecer la relación entre rendimiento académico, género y HHSS en escolares y universitarios (Oyarzún, Estrada, Pino, \& Oyarzun, 2012) o entre distintos perfiles de personalidad (Saldivia, Sandoval, Barría, Estrada \& Vinet, 2015). Por otro lado, Argentina ha sido uno de los países más prolíficos en esta temática en Latinoamérica, desde el desarrollo de instrumentos para su medición en distintos tramos de edad (Lacunza, Castro Solano, \& Contini, 2009; Lacunza \& Contini, 2009; Morán, Olaz, \& Del Prette, 2015; Olaz, Medrano, Greco, \& Del Prette, 2009;), así como análisis de su relación con autoestima (Cattan, 2005), variables sociodemográficas (Cohen, Esterkind, Lacunza, Valeria, \& Martinenghi, 2011) y familiares (Ison \& Rodriguez, 1997), adolescentes en contextos de pobreza (Contini, Coronel, Levin, \& Hormigo, 2012), una comparación entre géneros (Terán, Cabanillas, Morán, \& Olaz, 2014) o entre las HHSS de alumnos de primer y quinto año de psicología (Herrera et al., 2012); por otro lado, Ison (2011) ha evaluado la relación entre el entrenamiento de las HHSS y la disminución de 
conductas disruptivas en escolares.

Uno de los métodos de evaluación más utilizados para evaluar las HHSS en general y la eficacia de sus entrenamientos es el autoinforme, representando el $62.16 \%$ de los estudios instrumentales latinoamericanos (Morán \& Olaz, 2014). En otras áreas de la psicología, esta modalidad de evaluación también es una de las más utilizadas, representado por un $67 \%$ de las publicaciones instrumentales en psicología en general (Clark \& Watson, 2003). No obstante, desde hace más de una década, se ha venido alertando por la falta de instrumentos psicométricamente apropiados para la medición de las HHSS, existiendo en ese momento solo unos pocos con suficiente evidencia de validez y confiabilidad (Stravinsky \& Amado, 2001). En años más recientes, Morán y Olaz (2014) corroboraron que de los estudios instrumentales publicados sobre la medición de HHSS, solo el $8.11 \%$ realizaron análisis factorial confirmatorio $\mathrm{y}$ el $27.03 \%$ no aportaron evidencias sobre la estructura interna del instrumento. En este estudio también se constató que los países con mayor producción en materia de evaluación de este constructo son Brasil y Argentina, y solo el $2.6 \%$ de lo publicado es producción chilena.

Una de las poblaciones que más ha sido estudiada en América Latina son los estudiantes universitarios (Prytz Nilsson et al., 2014). Es probable que esto se deba a la mayor facilidad de acceso de este grupo para la investigación científica, pero por otro lado, en el caso específico de las HHSS, también puede deberse a que se trata de un conjunto vulnerable en materia de problemáticas interpersonales. El paso de la etapa de formación a la vida profesional supone un proceso de transición donde el estudiante no solo se encontrará con nuevos escenarios de actuación sino también con nuevas demandas para las cuales deberá poner en práctica los conocimientos adquiridos, así como también ciertas habilidades que le permitirán insertarse de manera competente en el mercado actual, caracterizado por niveles de exigencia cada vez más estrictos y competitivos, y donde no solo toman relevancia las cuestiones conceptuales propias de cada disciplina, sino también la capacidad de relacionarse con otros actores en estos nuevos espacios sociales de desempeño de la vida adulta (Z. A. P. Del Prette
\& A. Del Prette, 2003). Es en esta etapa que resulta importante que se consoliden los procesos de evolución de la empatía, el respeto por los demás, las relaciones sociales y el liderazgo, que tienen su inicio durante la adolescencia (Martorell, González, Ordoñez, \& Gómez, 2011)

En este sentido, los resultados de diversas investigaciones indican una alta frecuencia en estudiantes universitarios de problemas interpersonales tales como dificultades para interactuar con el sexo opuesto (Furnham, 1986) y ansiedad social (Collins, 2009), o por el contrario, desinhibición social (Camacho Acero, 2005); lo que estaría ligado a fracaso académico y otros problemas conductuales y/o de salud mental tales como depresión, ansiedad, distintos tipos de adicciones, trastornos sexuales, delincuencia y agresividad o problemas de pareja y familia (Arco Tirado, López Ortega, Heilborn Díaz, \& Fernández Martin, 2005; Buckner \& Schmidt, 2009; Cranford, Eisenberg, \& Serras, 2009; O'Grady, Cullum, Armeli, \& Tennen, 2011). Sumado a ello, estudios en población chilena evidencian que ciertos rasgos de personalidad disfuncionales, por ejemplo la sumisión, guardan relación con las HHSS específicamente en muestras que reciben tratamiento psicológico durante el primer año de cursado en la Universidad (Saldivia et al., 2015).

Existen diversos instrumentos en español para evaluar las HHSS en estudiantes universitarios. Muchos de ellos son adaptaciones de escalas construidas fuera de Latinoamérica. Específicamente en Chile, se reporta la validación de la Escala de Habilidades Sociales (EHS) para estudiantes universitarios de primer año (Miranda et al., 2014) cuya versión original se construyó para adultos en España (Gismero, 2000). Según los autores, este instrumento fue construido para evaluar HHSS y asertividad. Si bien la versión chilena de la escala presenta evidencias de validez de estructura interna y del test con variables externas, es importante considerar algunos puntos sobre otros aspectos de sus propiedades psicométricas.

Por ejemplo, dentro de los enunciados que componen los ítems de la EHS, algunos hacen referencia a comportamientos $y$ otros a sentimientos; no obstante, los desarrollos teóricos en su totalidad definen a las HHSS como un 
conjunto de conductas o comportamientos, lo que incluye la expresión de sentimientos (su componente conductual) pero no la vivencia emocional en sí (Caballo, 2007; Gresham, 2011; Hidalgo \& Abarca, 2000; Kelly, 2002; Olaz, 2012). En este sentido, incluir sentimientos sin conducta puede afectar su validez de contenido.

Por otro lado, al igual que la mayoría de los inventarios que evalúan HHSS, el formato de respuesta que emplea la EHS sigue un formato tipo Likert de cuatro puntos. La misma presenta alternativas de respuesta que indican indiferenciadamente cuánto la persona se identifica con el enunciado, su nivel de acuerdo con el mismo o la frecuencia con la que actúa o actuaria de la manera en que se presenta. Esta imprecisión en lo que se le pide al evaluado que responda genera información inespecífica producto de un error en el proceso de respuesta que se estimula y consecuentemente un posible sesgo en la validez y fiabilidad de la puntuación obtenida. Con respecto a esto último, en los resultados del estudio de Miranda et al. (2014) tres de las seis subescalas presentaron coeficientes de fiabilidad insatisfactorios (menores a .48).

Otro instrumento en español es el Cuestionario de Habilidades Sociales para Universitarios (CHS-U; Morán et al., 2015) construido en Argentina específicamente para evaluar el repertorio de HHSS en estudiantes universitarios. Este instrumento es una versión optimizada de la adaptación argentina del Inventario de Habilidades Sociales (Olaz et al., 2009). Está compuesto por ítems que presentan comportamientos ante situaciones sociales específicas y ante los cuales el evaluado debe responder con qué frecuencia actúa de la manera en que se describe. De esta forma la escala ofrece una medida válida de competencia social, ya que no se solicita una valoración sobre la calidad de las propias habilidades, sino de su frecuencia. Esto evita producir resultados sesgados, teniendo en cuenta que, según varios estudios empíricos, la autovaloración de las HHSS puede ser afectada por otros factores de naturaleza psicopatológica, tales como la depresión, la baja autoestima o la ansiedad social, caracterizada por un déficit de confianza en las propias habilidades independientemente de la calidad del desempeño real (Cartwright-Hatton, Tschernitz, \& Gomersall, 2005; Morán, 2017; Segrin, 1999).

El CHS-U fue construido exclusivamente para estudiantes universitarios hasta los 25 años, por lo que las situaciones sociales que incluye en sus ítems son características de esta población. El rango de edad de 20 a 25 años se le ha categorizado como adultez emergente o de transición a la adultez (Arnett, 2000), es decir, son jóvenes que no han dejado del todo estatus adolescente, pero tampoco poseen todos los atributos psicosociales de la etapa adulta; por lo general sienten ambigüedad hacia las actitudes y el funcionamiento adulto, pero tienen más independencia en la exploración de otros roles, pues pueden aún no estar necesariamente implicados en la formación de una familia, la búsqueda de empleo estable o la conyugalidad (Pizzmato, Calesso-Moreira, Cé, \& Eid, 2013)

Sumado a lo expuesto, el CHS-U también cuenta con diversos estudios que aportan evidencias de validez de contenido, de estructura interna, de convergencia, y ha presentado índices satisfactorios de fiabilidad (Morán et al., 2015). Con respecto a su estructura factorial, el cuestionario está constituido por cinco dimensiones. El primer factor se denomina Habilidades Sociales Académicas, haciendo referencia a habilidades necesarias para un adecuado desempeño académico, por ejemplo hacer preguntas y hablar en púbico en un entorno educativo formal. El segundo factor evalúa Habilidades de Oposición Asertiva, necesarias para oponerse o rechazar la conducta o comentarios inaceptables de otra persona y para conseguir una conducta más aceptable en el futuro. El tercer factor mide Habilidades Sociales Empáticas y de expresión de sentimientos positivos, necesarias para transmitir calidez y expresar cumplidos, elogios, aprecio, y sentimientos personales y opiniones a los demás cuando la conducta positiva de estos lo justifica. También se incluye dentro de esta dimensión el ofrecimiento de conducta positiva recíproca para corresponder lo que ha hecho el otro, aceptar los propios errores, disculparse y brindar apoyo. El factor cuatro evalúa Habilidades para el Abordaje Afectivo-sexual, que posibilitan al individuo contactar y iniciar relaciones con personas que despiertan algún interés sexual o erótico, 
permitiendo a la persona entablar conversaciones y establecer encuentros con personas que le atraen. Finalmente el quinto factor se denomina Habilidades Conversacionales, necesarias para iniciar y mantener conversaciones informales con otros de manera fluida sin experimentar demasiada ansiedad. Estos cinco tipos de habilidades responden a la teoría de Kelly (2002), han sido evidenciados en la Escala de Autoeficacia Social para estudiantes universitarios (Olaz. 2012) y constituyen importantes destrezas para el desenvolvimiento social general de los estudiantes de nivel superior (Blasco, 2016; García \& Torres, 2016; Moreno, Segura, \& Torres, 2013; Noguera, Martí-Vilar, \& Almerich, 2014; Terán et al., 2014; Tobón, Zapata, Lopera, $\&$ Duque, 2014).

En los últimos años se ha observado un aumento de la movilidad de estudiantes universitarios entre Chile y Argentina. Según cifras oficiales, un $4.6 \%$ de todos los extranjeros cursando estudios superiores en Chile son de nacionalidad argentina (Salamanca, Sazo, \& Rolando, 2015); a su vez, sólo en la Universidad de Buenos Aires se reportaron casi 900 estudiantes de nacionalidad chilena (Bazán, 2015). Teniendo en cuenta las características mencionadas y la necesidad de contar con un instrumento alternativo a la EHS para la evaluación de las HHSS en la comunidad universitaria de Chile, el objetivo de este trabajo es realizar un estudio de validación transcultural del CHS-U en estudiantes chilenos y argentinos.

\section{Método}

\section{Diseño}

Se utilizó un diseño instrumental, de tipo trasversal.

\section{Participantes}

Participaron 1025 estudiantes universitarios, 771 argentinos y 254 chilenos. Del total, 627 fueron mujeres $(61.2 \%)$ y 398 hombres $(38.8 \%)$. La media total de edad fue de 22.05 años $(D T=1.66)$ con un rango desde los 20 a los 25 años. Una comparación entre los dos grupos nacionales se puede observar en la Tabla 1 .

\section{Instrumentos}

Se utilizó el Cuestionario de Habilidades Sociales para Universitarios [CHS-U], elaborado por Morán et al. (2015). Este cuestionario cuenta con 20 reactivos, en una Escala Likert de cinco puntos que van desde 1 (nunca o de vez en cuando) a 5 (siempre o casi siempre). Los ítems se agrupan formando una estructura de cinco factores. El primer factor se denomina Habilidades Sociales Académicas (1-4), por ejemplo, "Si encuentro que me han corregido mal un examen, le solicito al profesor que lo revise". El segundo factor se denomina Habilidades de Oposición Asertiva (5-8), por ejemplo, "Si en un comercio me tratan mal comunico mi disconformidad al encargado". El tercer factor se trata de Habilidades Sociales Empáticas y de expresión de sentimientos positivos (9-12), por ejemplo, "Si un amigo se siente mal por algo que dije o hice, le pido disculpas". El cuarto factor son Habilidades para el abordaje Afectivo-Sexual (13-16), por ejemplo, "Al sentir deseos de conocer a alguien a quien no fui presentado(a), yo mismo me presento". Por último, el quinto factor son Habilidades conversacionales (17-20), por ejemplo, "Mientras estoy en la fila de un banco inicio conversaciones con otras personas". Se estableció validez de constructo con análisis factorial exploratorio y confirmatorio, y presenta una confiabilidad compuesta entre .75 y .90 en todas las escalas.

Además se incluyó un cuestionario sociodemográfico con preguntas relativas al género, edad, carrera y nacionalidad.

\section{Procedimiento}

Los participantes argentinos fueron seleccionados utilizando un muestreo no aleatorio de tipo accidental. Se acudió a ocho universidades de la Provincia de Córdoba y se accedió a recolectar los datos en los cursos que aceptaron

Tabla 1. Comparación entre participantes chilenos y argentinos en edad y proporción de hombres y mujeres

\begin{tabular}{lcccccc}
\hline & $N$ & Rango & $M$ & $D E$ & \% Hombres & \% Mujeres \\
\hline Población Chilena & 254 & $20-25$ & 22.30 & 1.72 & 34.3 & 65.7 \\
Población Argentina & 771 & $20-25$ & 21.96 & 1.64 & 40.3 & 59.7 \\
Población Total & 1025 & $20-25$ & 22.05 & 1.66 & 38.8 & 61.2 \\
\hline
\end{tabular}


participar. En total se recolectaron datos de alumnos de 56 carreras de diferentes facultades. Los participantes chilenos fueron seleccionados a través de un muestreo aleatorio de conglomerado en dos etapas. Para ello, a partir de dos universidades de la ciudad de Concepción que aceptaron participar, se seleccionaron aleatoriamente cinco carreras en cada una de ellas, y dentro de las carreras se seleccionó aleatoriamente un curso para ser encuestado.

Para ambas muestras, de los cursos seleccionados, fueron incluidas las personas cuyas edades fluctuaran entre 20 y 25 años. Los participantes fueron encuestados por estudiantes de psicología, previamente capacitados. Los cuestionarios fueron auto-aplicados en presencia del encuestador, previa firma de una carta de consentimiento informado en el cual se garantizaba la confidencialidad, resguardo de los datos entregados, y participación voluntaria. No hubo personas que se negaran a responder.

\section{Análisis de datos}

En primera instancia, se realizó un análisis factorial confirmatorio con el total de la muestra. Para ello se realizó previamente un análisis del supuesto de normalidad multivariante a través del coeficiente de Mardia, utilizando como parámetro un valor inferior a 70, límite máximo recomendado por Rodríguez y Ruiz (2008) para utilizar el método de estimación de máxima verosimilitud, preferible sobre otros al permitir el contraste de hipótesis de las estimaciones. Se utilizaron los siguientes índices y criterios de ajuste (Hu \& Bentler, 1999; $\mathrm{Yu}, 2002)$ : a) $\chi 2$ : valores no significativos indican un buen ajuste, b) $\chi 2 / g l:$ un buen ajuste está indicado por valores menores a 2, c) CFI y TLI: un ajuste aceptable está dado por valores $\geq .90$, un buen ajuste está indicado por valores $\geq .95$, d) RMSEA: un ajuste aceptable está indicado por valores $\leq .08(90 \% \mathrm{CI}$ $\leq .10)$, un buen ajuste está indicado por valores $\leq .05(90 \% \mathrm{CI} \leq .08)$.

Luego se estudió la invarianza entre los participantes argentinos y chilenos a través de análisis multimuestra. Tradicionalmente, la prueba de invarianza entre niveles se realiza estudiando las diferencias en el estadístico Chi-Cuadrado entre un nivel y otro, ya que los modelos se encuentran anidados (Bollen \& Long, 1994).
Como este estadístico es sensible al tamaño de la muestra, es más probable que sea rechazada la invarianza en muestras grandes, aunque en términos prácticos las diferencias sean mínimas. Por tal motivo, en este estudio se considera principal indicio de invarianza que el CFI no disminuya más de .01 $(\Delta \pm .01)$ con respecto al modelo anterior (Cheung \& Rensvold, 2002). Se espera además un TLI superior a .90 , y un RMSEA inferior a .08 y ausencia de diferencias importantes entre cada modelo anidado.

Finalmente, se realizaron los análisis descriptivos de la escala total y de cada uno de sus factores.

Para todos estos análisis se utilizó el software estadístico SSPS Statistics 23 y el Amos v18 de IBM.

\section{Resultados}

Se procede a realizar un Análisis Factorial Confirmatorio del modelo de cinco factores (Morán et al., 2015). El coeficiente de Mardia obtuvo un valor de 22.80. Si bien se encuentra fuera del rango \pm 5 sugerido por Bentler (2005) para asumir una normalidad multivariante, está por debajo del valor crítico de \pm 70 recomendado para el uso del método de estimación de máxima verosimilitud (Rodríguez \& Ruiz, 2008).

Los índices de ajuste obtenidos fueron: $\chi^{2}=525.02, \mathrm{p}<.001 ; \chi^{2} / \mathrm{gl}=3.28 ; \mathrm{TLI}=.91 ; \mathrm{CFI}=.92 ;$ RMSEA $=.05$ (90\% IC, .04 -.05). Estos índices muestran que el modelo ajusta bien a los datos, confirmando la presencia de los cinco factores. En la Figura 1 se puede observar el modelo evaluado.

Una vez confirmada la estructura factorial de cinco dimensiones, se procedió a evaluar la invarianza factorial del modelo entre los participantes argentinos y chilenos. Para ello, se realizó la evaluación secuencial de la invarianza configural, métrica, fuerte y estricta (Elosúa, 2005). La invarianza configural exige que los factores queden especificados por los mismos ítems en las dos poblaciones; el rechazo de la hipótesis de invarianza configural implica la falta de equivalencia sustantiva de constructos entre las poblaciones. La invarianza métrica examina la igualdad de coeficientes de regresión. La invarianza fuerte examina la igualdad en los interceptos. La invarianza estricta examina la 


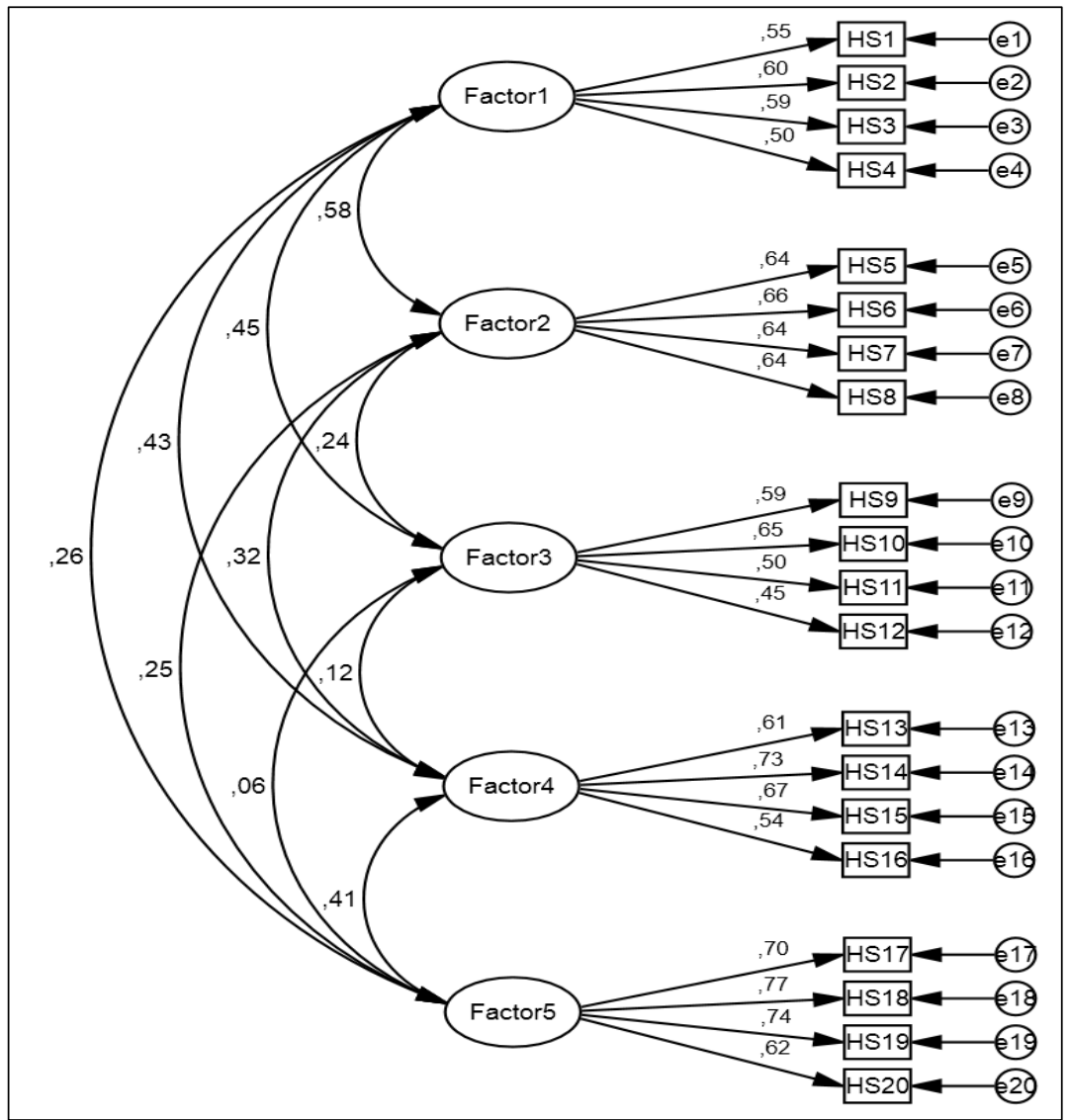

Figura 1. Modelo de 5 factores del Cuestionario de Habilidades Sociales. Los valores están estandarizados

Tabla 2. Modelos de invarianza factorial entre participantes chilenos y argentinos

\begin{tabular}{lcccccc}
\hline Modelos & $\chi^{2}(g l)$ & $\Delta \chi^{2}$ & CFI & $\Delta$ CFI & TLI & RMSEA \\
\hline M1: configural & $741.07(320)$ & - & .91 & - & .90 & .04 \\
M2: métrica & $798.92(335)$ & -57.85 & .91 & -.00 & .89 & .04 \\
M3: fuerte & $1156.31(355)$ & -357.39 & .84 & -.07 & .83 & .05 \\
M4: estricta & $1393.72(375)$ & -237.41 & .79 & -.05 & .79 & .05 \\
\hline
\end{tabular}

Nota: $\mathrm{M} 1=\sin$ restricciones; $\mathrm{M} 2=\mathrm{M} 1+$ igualdad en coeficientes factoriales;

$\mathrm{M} 3=\mathrm{M} 2+$ igualdad en los interceptos; M4=M3+igualdad en varianza y covarianza de errores.

igualdad en la varianza y covarianza de los errores y es el mayor nivel de acuerdo alcanzable entre estructuras factoriales.

El análisis entre los dos grupos muestra la existencia de invarianza configural, pues los valores de los índices de ajuste RMSEA, TLI y CFI fueron aceptables. También muestra invarianza métrica, dado que el CFI disminuyó su valor en .00 con respecto al modelo anterior y los otros índices casi no sufrieron variaciones, por lo que se puede concluir que las cargas factoriales son equivalentes en las dos submuestras. La invarianza fuerte y estricta no son aceptables pues los índices de ajuste no son apropiados y el CFI disminuye en un valor superior a .01 , por lo que es posible concluir que los dos modelos evaluados no son equivalentes respecto a los interceptos y los residuos (ver Tabla 2).

Los datos descriptivos de cada subescala del CHS-U y de la escala total se muestran en la tabla 3. Se observa que el alfa de Cronbach de la escala total es adecuada, presentando una correlación ítem-test que fluctúa de $\mathrm{r}=.27$ a .50 . 
Tabla 3. Estadísticos descriptivos y alfa de Cronbach de escala CHS-U y subescalas (N=1025)

\begin{tabular}{|c|c|c|c|c|c|c|c|c|c|c|c|c|c|c|c|c|c|}
\hline & \multirow[b]{3}{*}{ Min } & \multirow[b]{3}{*}{ Máx } & \multirow[b]{3}{*}{ M } & \multirow{2}{*}{\multicolumn{4}{|c|}{ Total }} & \multicolumn{5}{|c|}{ Sexo } & \multicolumn{5}{|c|}{ Nacionalidad } \\
\hline & & & & & & & & \multicolumn{2}{|c|}{ Mujeres } & \multicolumn{2}{|c|}{ Hombres } & \multirow{2}{*}{$\mathrm{t}$} & \multicolumn{2}{|c|}{ Chilena } & \multicolumn{2}{|c|}{ Argentina } & \\
\hline & & & & $\mathrm{DE}$ & Asim. & Curt. & $\alpha$ & M & DT & M & DT & & M & DT & M & DT & \\
\hline $\begin{array}{l}\text { HH.SS. } \\
\text { Académicas }\end{array}$ & 5 & 20 & 14.80 & 3.10 & -0.40 & -0.20 & 0.64 & 14.69 & 3.10 & 14.97 & 3.11 & -1.42 & 14.94 & 3.18 & 14.75 & 3.08 & 0.85 \\
\hline $\begin{array}{l}\text { HH.SS. Oposición } \\
\text { Asertiva }\end{array}$ & 4 & 20 & 11.16 & 4.10 & 0.26 & -0.66 & 0.74 & 11.11 & 4.14 & 11.25 & 4.03 & -0.55 & 12.94 & 4.34 & 10.58 & 3.84 & $7.76^{* * *}$ \\
\hline HH.SS. Empática & 5 & 20 & 16.05 & 2.76 & -0.67 & 0.35 & 0.62 & 16.16 & 2.74 & 15.87 & 2.80 & 1.61 & 16.77 & 2.82 & 15.81 & 2.70 & $4.86^{* * *}$ \\
\hline $\begin{array}{l}\text { HH.SS. Abordaje } \\
\text { Afectivo Sexual }\end{array}$ & 4 & 20 & 10.51 & 3.82 & 0.28 & -0.61 & 0.73 & 9.44 & 3.58 & 12.19 & 3.58 & $-11.98 * * *$ & 10.12 & 4.13 & 10.64 & 3.71 & -1.76 \\
\hline $\begin{array}{l}\text { HH.SS. } \\
\text { Conversacionales }\end{array}$ & 4 & 20 & 9.91 & 3.82 & 0.40 & -0.60 & 0.80 & 9.96 & 3.85 & 9.84 & 3.76 & 0.49 & 8.65 & 4.19 & 10.33 & 3.59 & $-5.73 * * *$ \\
\hline Escala Total & 28 & 100 & 62.43 & 10.96 & 0.26 & 0.23 & 0.80 & 61.35 & 10.84 & 64.12 & 10.94 & $-3.98 * * *$ & 63.43 & 12.14 & 62.10 & 10.53 & 1.56 \\
\hline
\end{tabular}

$* * * p<.001$

\section{Discusión}

Las habilidades para las interacciones interpersonales tienen relación directa con el éxito personal y social, así como también con su éxito y competencia profesional. Las mismas tienen tanta importancia como las habilidades técnicas, cognitivas e intelectuales que pueden adquirirse durante el periodo de formación (García Rojas, 2010; Hargie \& Marshall, 1986; Marín Sánchez \& León Rubio, 2001; Mendo-Lázaro, León, FelipeCastaño, Polo, \& Palacios-García, 2016). Por ello, el estudio de las HHSS ha mostrado un fuerte incremento en los últimos años, lo que ha sido acompañado de estudios instrumentales que han aportado herramientas para la evaluación del constructo en las principales poblaciones estudiadas, tales como niños, adolescentes y estudiantes universitarios.

A pesar de que en Latinoamérica se evidencia una interesante producción de instrumentos de constructos relacionados con las HHSS, como la asertividad, la autoeficacia social o la competencia social (Morán \& Olaz, 2014), en Chile no se reportan escalas para estudiantes universitarios a excepción de la ya comentada EHS (Miranda et al., 2014), la cual si bien constituye un importante aporte para el estudio de esta temática, evidencia algunos problemas psicométricos como la baja confiabilidad en algunos de sus factores. Ante la necesidad de incrementar el desarrollo de este campo en población chilena, el presente trabajo tuvo como objetivo la validación transcultural del
CHS-U (Morán et al., 2015) entre estudiantes universitarios de Chile y Argentina.

Como resultado, se obtuvo una escala compuesta por los 20 ítems originales de la escala distribuidos en una estructura factorial de cinco dimensiones evidenciada mediante análisis factorial confirmatorio. Los índices de ajuste obtenidos fueron satisfactorios (TLI=.91; CFI=.92; RMSEA=.05) corroborando que el instrumento presenta la misma estructura encontrada por sus autores en los estudios de construcción y validación.

Con respecto a la fiabilidad de la escala, los coeficientes de consistencia interna fueron adecuados para la mayoría de los factores y para la escala general. Sin embargo en los factores uno $\mathrm{y}$ tres los valores fueron moderados $\mathrm{y}$ considerados como críticos por la literatura para su empleo en diagnósticos e intervenciones (Carretero-Dios \& Pérez, 2007). No obstante, con valores cercanos a .70 la fiabilidad puede considerarse adecuada para su uso en investigación (Nunnally \& Bernstein, 1995).

En la evaluación de la invarianza factorial entre los participantes argentinos y chilenos, observamos que existe equivalencia en los grupos tanto a nivel configural como a nivel métrico. A nivel configural significa que las dos muestras presentan equivalencia en relación a la dimensionalidad de la escala, en otras palabras, las diferentes HHSS pueden agruparse en cinco factores en ambas culturas, lo que resulta fundamental cuando se efectúan investigaciones 
de carácter transcultural. A nivel métrico implica que hay equivalencia en las varianzas factoriales y pendientes de regresión, requisito considerado suficiente para comparar grupos para muchos investigadores (Vandenberg, 2002). Por otro lado, no se confirma la invarianza fuerte, es decir, los grupos mostraron no ser equivalentes en los valores de sus interceptos, lo que implica que no existe paridad entre los orígenes de la escala de medida de las variables latentes. El último nivel de restricción, la invarianza estricta, era esperable que no se alcanzara dada la ausencia de paridad en el modelo de invarianza fuerte.

La presencia de invarianza en la dimensionalidad y cargas factoriales de la escala se explica dado que las dos culturas, argentina y chilena, son semejantes y próximas entre sí tanto geográficamente (son países vecinos), como por razones socio-culturales, pues comparten idioma y costumbres, entre otras características. Frente a esto, es esperable que el modelo se comporte de forma invariante, aun cuando exista un funcionamiento no-invariante en algunos interceptos.

El estudio contiene algunas limitaciones que es necesario mencionar. En primer lugar, la muestra argentina no es aleatoria, lo que puede afectar la generalización de sus resultados. En segundo lugar, no se realizaron pruebas de validez de criterio; tampoco se evaluó la estabilidad temporal al constituir este estudio una sola medición de carácter transversal.

Es necesario tener en cuenta algunas sugerencias para continuar analizando las propiedades psicométricas de la escala tales como llevar a cabo estudios con muestras chilenas más amplias y que contemplen el análisis de la estabilidad de la escala, de sus relaciones con medidas externas, la validez de grupos contrastados por condición clínica - no clínica y la obtención de evidencia de la sensibilidad experimental del instrumento a partir de una intervención. No obstante, los resultados obtenidos constituyen una evidencia preliminar de validez y confiabilidad para CHS-U, el cual puede utilizarse inicialmente para investigación, permitiendo abrir y ampliar el campo de estudio de este constructo en los estudiantes universitarios chilenos.
En ese contexto, el CHS-U significa un aporte pues se constituye como un instrumento alternativo al EHS, con adecuados indicadores psicométricos y claridad conceptual, que permitirá evaluar sin sesgos tanto a estudiantes chilenos como argentinos.

\section{Referencias}

Abarca, N., \& Hidalgo C. G. (1989). Evaluación psicométrica de habilidades sociales en jóvenes universitarios chilenos. Revista Análisis del Comportamiento, 4, 51-62.

Arco Tirado, J. L, López Ortega, S., Heilborn Díaz, V., \& Fernández Martin, F. (2005). Terapia breve en estudiantes universitarios con problemas de rendimiento académico y ansiedad: Eficacia del modelo "La Cartuja". International Journal of Clinical and Health Psychology, 5(3), 589-608.

Arnett, J. (2000). Emerging adulthood: A theory of development from the late teens through the twenties. American Psychologist, 55, 469480. doi: 10.1093/acprof:oso/9780199929382. 001.0001

Bazán, I. (2015, enero 04). La promesa a medias de las universidades transandinas. Diario $\mathrm{La}$ Tercera, Chile. Disponible en: http://diario.latercera.com/2015/01/04/01/cont enido/reportajes/25-180930-9-la-promesa-amedias-de-las-universidades-transadinas.shtml

Bentler, P. (2005). EQS Structural equations program manual. Encino, CA: Multivariate Software.

Blasco, M. L. M. (2016). Estudio de las habilidades sociales o de la conducta asertiva: En el alumnado de la Escuela universitaria de enfermería de Huesca. Revista ROL de Enfermería, 39(9), 36-43.

Bollen, K., \& Long, J.S. (1994). Testing structural equation models. Newbury Park, CA: Sage.

Buckner, J. D., \& Schmidt, N. B. (2009). Understanding social anxiety as a risk for alcohol use disorders: Fear of scrutiny, not social interaction fears, prospectively predicts alcohol use disorders. Journal of Psychiatric Research, 43(4), 477-483. doi: 10.1016/j.jpsychires.2008.04.012

Bunford, N., Evans, S. W., Becker, S. P., \& Langberg, J. M. (2015). Attention- 
deficit/hyperactivity disorder and social skills in youth: A moderated mediation model of emotion dysregulation and depression. Journal of Abnormal Child Psychology, 43(2), 283-296. doi: 10.1007/s10802-014-9909-2

Caballo, V. (2007). Manual de evaluación y entrenamiento de las habilidades sociales (7ma Ed.). Madrid: Siglo XXI.

Camacho Acero, I. (2005). Consumo de alcohol en universitarios: Relación funcional con los factores sociodemográficos, las expectativas y la ansiedad social. Acta Colombiana de Psicología, 8(1), 91-120.

Carretero-Dios, H., \& Pérez, C. (2007). Normas para el desarrollo y revisión de estudios instrumentales: Consideraciones sobre la selección de tests en la investigación psicológica. International Journal of Clinical and Health Psychology, 7, 863-882

Cattan, A. (2005). Estudio comparativo de autoestima y habilidades sociales en pacientes adictos de una comunidad terapéutica. Salud y Drogas, 5(1), 139-164.

Cartwright-Hatton, S., Tschernitz, N., \& Gomersall, H. (2005). Social anxiety in children: social skills deficit, or cognitive distortion? Behaviour Research and Therapy, 43(1), 131-141. doi: 10.1016/j.brat.2003.12.00 3

Cheung, G., \& Rensvold, R. (2002). Evaluating goodness-of-fit indexes for testing measurement invariance. Structural Equation Modeling, 9, 233-255. doi: 10.1207/S1532800 7SEM0902_5

Clark, L. A., \& Watson, D. (2003). Constructing validity: Basic issues in objective scale development. En A.E. Kazdin (Ed.), Methodological issues \& strategies in clinical research (3ra Ed., pp. 207-231). Washington: APA.

Cohen, S., Esterkind, A., Lacunza, A., Valeria, S., \& Martinenghi, C. (2011). Habilidades sociales y contexto sociocultural. Un estudio con adolescentes a través del BAS-3. Revista Iberoamericana de Diagnóstico y Evaluación - e Avaliação Psicológica, 29(1), 167-185.

Collins, A. B. (2009). Investigation of social anxiety prevalence and anxiety sensitivity among college students. Reno, EEUU: Universidad de Nevada.
Contini, N., Coronel, C. P., Levin, M., \& Hormigo, K. (2012). Las habilidades sociales en contextos de pobreza. Un estudio preliminar con adolescentes de la Provincia de Tucumán. Perspectivas en Psicología, 7(1), 112-120.

Cranford, J. A., Eisenberg, D., \& Serras, A. M. (2009). Substance use behaviors, mental health problems, and use of mental health services in a probability sample of college students. Addictive Behaviors, 34, 134-145. doi: 10.1016/j.addbeh.2008.09.004

Da Costa, C. S. L., \& Del Prette, A. (2012). Estudo comparativo de observação de habilidades sociais de gêmeas com e sem deficiência visual. Revista Educação Especial, 25(42), 75-88. doi: 10.5902/198468 6x3552

Del Prette A., \& Del Prette, Z.A.P. (2003). Habilidades sociais, desenvolvimento e aprendizagem: Questões conceituais, avaliação e intervenção. Campinas: Alínea.

Del Prette, A., \& Del Prette, Z.A.P. (2008). Psicologia das relações interpessoais e habilidades sociais: Vivências para o trabalho em grupo (6ta Ed.). Petrópolis: Vozes

Del Prette, A., \& Del Prette, Z.A.P. (2014). Psicologia das habilidades sociais: Diversidade teórica e suas implicações (2da Ed.) (pp. 17-66). Petrópolis: Vozes.

Del Prette, Z. A. P., \& Del Prette, A. (2002). Psicología de las Habilidades Sociales. Terapia y educación. México: El Manual Moderno.

Del Prette, Z. A. P., \& Del Prette, A. (2003). No contexto da travessia para o ambiente de trabalho: Treinamento de habilidades sociais com universitários. Estudos de Psicología, $8(3)$, 413-420. doi: 10.1590/s1413294x2003000300008

Del Prette, Z. A. P., \& Del Prette, A. (2005). Psicología de las habilidades sociales. Terapia y educación. México: El Manual Moderno.

Del Prette, Z. A. P., \& Del Prette, A. (2011). Psicologia dashabilidades sociais na infância. ( $5^{\mathrm{a}}$ Ed). Petrópolis: Vozes.

Del Prette, A., Del Prette, Z. A. P., \& Barreto, M. C. M. (1999). Habilidades sociales en la formación del psicólogo: Análisis de un 
programa de intervención. Psicología Conductual, 7, 27-47.

Deming, D. J. (2015). The growing importance of social skills in the labor market. NBER Working Paper, 21473. doi: 10.3386/w21473

Elosúa, P. (2005). Evaluación progresiva de la invarianza factorial entre las versiones original y adaptada de una escala de autoconcepto. Psicothema, 17(2), 356-362.

Furnham, A. (1986). Social skills training with adolescents and young adults, En P. Hollin \& C.R. Trower (Eds,). Handbook of social skills training (pp. 33-57). Oxford: Pergamon Press.

García, G. S., \& Torres, C. L. (2016). Relaciones de pareja en las/os estudiantes universitarios/as y sus implicancias en sus interacciones sociales, TACNA 2011. Revista Peruana de Obstetricia y Enfermería, 10(1), 1-9.

García Rojas, A. D. (2010). Estudio sobre la asertividad y las habilidades sociales en el alumnado de Educación Social. Revista de Educación, 12, 225-239.

Gil Rodríguez, F., Cantero Sánchez, F. J., \& Antino, M. (2013). Tendencias actuales en el ámbito de las habilidades sociales. Apuntes de Psicología, 31(1), 51-57.

Gismero, E. (2000). EHS Escala de habilidades sociales. Madrid: TEA Publicaciones de Psicología Aplicada.

Gresham, F. M. (2011). Análise do comportamento aplicada às habilidades sociais. En A. Del Prette y Z.A.P. Del Prette (Orgs.). Psicologia das habilidades sociais: Diversidade teórica e suas implicações (2da Ed.) (pp. 17-66). Petrópolis: Vozes.

Gutiérrez Carmona, M., \& Expósito López, J. (2015). Autoconcepto, dificultades interpersonales, habilidades sociales y conductas asertivas en adolescentes. REOP Revista Española de Orientación y Psicopedagogía, 26(2), 42-58. doi: 10.5944/reop.vol.26.num.2.2015.15215

Herrera, A., Freytes, V., López, G., \& Oláz, F. (2012). Un estudio comparativo sobre las habilidades sociales en estudiantes de Psicología. International Journal of Psychology and Psychological Therapy, 12(2), 277-287.
Hidalgo, C., \& Abarca, N. (2000). Comunicación interpersonal Programa de entrenamiento de habilidades sociales. Santiago, Chile: Universidad Católica de Chile.

Hargie, O., \& Marshall, P. (1986). Interpersonal communication: A theoretical framework. En O. Hargie, A handbook of communication skills (pp. 29-63). Londres: Croom Helm.

Hu, L., \& Bentler, P. (1999). Cutoff criteria for fit indexes in covariance structure analysis: Conventional criteria versus new alternatives. Structural Equation Modeling, 6, 1-55. doi: 10.1080/10705519909540118

Ison, M. (2001). Training in social skills: An alternative technique for handling disruptive child behavior. Psychological Reports, 88(3), 903-911. doi: 10.2466/PR0.88.3.903-911

Ison, M., \& Rodríguez, I. (1997). Desarrollo de habilidades sociales en el tratamiento de conductas problemas infantiles. Revista Mexicana de Psicología, 14 (2), 129-137.

Kelly, J. (2002). Entrenamiento de las Habilidades Sociales. $\left(7^{\mathrm{a}}\right.$ Ed.) España: Descleé de Brower.

Lacunza, A., \& Contini, N. (2009). Las habilidades sociales en niños preescolares en contextos de pobreza. Ciencias Psicológicas, 3(1), 57-66.

Lacunza, A., Castro Solano, A., \& Contini, N. (2009). Habilidades sociales preescolares: Una escala para niños en contextos de pobreza. Revista de Psicología (PUCP), 27, 3-25.

Marín Sánchez, M., \& León Rubio, J. M. (2001). Entrenamiento en habilidades sociales: Un método de enseñanza-aprendizaje para desarrollar las habilidades de comunicación interpersonal en el área de enfermería. Psicothema, 13 (2), 247-251.

Martorell, C., González, R., Ordóñez, A., \& Gómez, O. (2011). Estudio confirmatorio del Cuestionario de Conducta Prosocial (CCP) y su relación con variables de personalidad y socialización. Revista Iberoamericana de Diagnóstico y Evaluación - e Avaliação Psicológica, 2(32), 35-52.

Mendo-Lázaro, S., León, B., Felipe-Castaño, E., Polo, M. I., \& Palacios-García, V. (2016). Evaluación de las habilidades sociales de estudiantes de Educación Social. Revista de 
Psicodidáctica, $\quad 21(1), \quad$ 139-156. $\quad$ doi: 10.1387/revpsicodidact.14031

Miranda, E., Riquelme, E., Cifuentes, H., \& Riquelme, P. (2014). Análisis factorial confirmatorio de la Escala de habilidades sociales en universitarios chilenos. Revista Latinoamericana de Psicología, 46(2), 73-82. doi: 10.1016/s0120-0534(14)70010-x

Morán, V. E. (2017). Modelo de ansiedad social en estudiantes universitarios. (Tesis doctoral no publicada). Universidad Nacional de Córdoba, Argentina.

Morán, V. E., \& Olaz, F. O. (2014). Instrumentos de evaluación de habilidades sociales en América Latina: Un análisis bibliométrico. Revista de Psicología, 23(1), 93-105. doi: 10.5354/0719-0581.2014.32877

Morán, V. E., Olaz, F. O., \& Del Prette, Z.A.P. (2015). Social Skills Questionnaire for Argentinean College Students (SSQ-U) Development and Validation. The Spanish Journal of Psychology, 18, E95. doi: 10.1017/sjp.2015.92

Moreno, M. R. B., Segura, M. D., \& Torres, M. Á. G. (2013). Entrenamiento en habilidades sociales en estudiantes universitarios de Magisterio de la especialidad de Educación Primaria: Un estudio preliminar. Apuntes de Psicología, 31(1), 85-91.

Noguera, J. J. M., Martí-Vilar, M., \& Almerich, G. (2014). Responsabilidad social universitaria: Influencia de valores y empatía en la autoatribución de comportamientos socialmente responsables. Revista Latinoamericana de Psicología, 46(3), 160168. doi: 10.1016/s0120-0534(14)70019-6

Nunnally, J. C., \& Bernstein, I. J. (1995). Teoría psicométrica. Madrid: McGraw-Hill.

O'Grady, M. A., Cullum, J., Armeli, S., \& Tennen, H. (2011). Putting the relationship between social anxiety and alcohol use into context: A daily diary investigation of drinking in response to embarrassing events. Journal of Social and Clinical Psychology, 30(6), 599-615. doi: 10.1521/jscp.2011.30.6.5 99

Olaz, F. O. (2012) Escala de Autoeficacia Social para Universitarios (EAS-U) Estudios Psicométricos y Bases Conceptuales. España: Editorial Académica Española.
Olaz, F. O., Medrano, L, Greco, M. E., \& Del Prette, Z. A. P. (2009). Argentinean Adaptation of the Social Skills Inventory IHSDel-Prette. Spanish Journal of Psychology, 12(2), 756-766. doi: 10.1017/s113874160000 2122

Olaz, F. O., \& Morán, V. E. (2014). Habilidades sociales en la formación y ejercicio de la psicoterapia. Saarbrücken: Editorial Académica Española.

Olivares, J., Olivares-Olivares, P. J., \& Maciá, D. (2014). Entrenamiento en habilidades sociales y tratamiento de adolescentes con fobia social generalizada. Psicología Conductual, 22(3), 441-459.

Oyarzún, G., Estrada, C., Pino, E., \& Oyarzun, M. (2012). Habilidades sociales y rendimiento académico: Una mirada desde el género. Acta Colombiana de Psicología, 15(2), 21-28

Pedraza, L., Socarrás, X., Fragozo, A., \& Vergara, M. (2014). Caracterización de las habilidades sociales en estudiante de psicología de una universidad pública del Distrito de Santa Marta. Tesis Psicológica, 9(2), 190-201.

Pizzmato, A., Calesso-Moreira, M., Cé, J., \& Eid, A. (2013). Inmigración y maternidad en la transición a la vida adulta en jóvenes Latinoamericanas. Psicología desde el Caribe, 30(2), 236-256.

Prytz Nilsson, N., Suárez, A. B., \& Olaz, F. O. (2014). Estudio bibliométrico de las publicaciones científicas sobre el área de Habilidades Sociales en América Latina. (2014). En F. O Olaz. \& V. E. Morán (Eds.). Habilidades Sociales en la Formación y Ejercicio de la Psicoterapia. Saarbrücken: Editorial Académica Española.

Reche, M. P. C., \& Lucena, F. J. H. (2016). El entrenamiento en habilidades sociales y su incidencia en el desarrollo psicoafectivo del alumno. REXE-Revista de Estudios y Experiencias en Educación, 4(8), 63-90.

Rodríguez, M., \& Ruíz, M. (2008). Atenuación de la asimetría y de la curtosis de las puntuaciones observadas mediante transformaciones de variables: Incidencia sobre la estructura factorial. Psicológica, 29(2), 205-227.

Salamanca, J., Sazo, P., \& Rolando, R. (2015). Situación de la matrícula de extranjeros en 
educación superior en Chile: Datos 2014. Santiago, Chile: Ministerio de Educación: Recuperado de http://www.mifuturo.cl/image s/Estudios/Estudios_SIES_DIVESUP/informe _estudiantes_extranjeros_2014.pdf

Saldivia, P., Sandoval, M., Barría, M., Estrada, C., \& Vinet, E. (2015). Asociación entre perfiles del MACI y habilidades sociales en población adolescente normal y consultante. Revista Iberoamericana de Diagnóstico y Evaluación - e Avaliação Psicológica, 1(39), 4-21.

Segrin, C. (1999). Social skills, stressful life events, and the development of psychosocial problems. Journal of Social and Clinical Psychology, 18(1), 14-34. doi: 10.1521/jscp.1999.18.1.14

Sofronoff, K. (2016). 32.0 Social skills training approaches for children and youth: Theory, research, and practice. Journal of the American Academy of Child \& Adolescent Psychiatry, 55(10), S49. doi: 10.1016/j.jaac.2016.07.628

Stravinsky, A., \& Amado, D. (2001). Social phobia as a deficit in social skills. In: S. Hoffman \& P. DiBartolo (Eds.), From social anxiety to social phobia: Multiple perspectives (pp. 107-129). Boston: Allyn \& Bacon.

Terán, M. G., Cabanillas, G. A., Morán, V. E., \& Olaz, F. O. (2014). Diferencias de género en habilidades sociales en estudiantes universitarios de Argentina. Disertaciones: Anuario electrónico de estudios en Comunicación Social, 7(2), 114-135.

Tobón, O. E. A., Zapata, S. J. C., Lopera, I. C. P., \& Duque, J. W. S. (2014). Formación académica, valores, empatía y comportamientos socialmente responsables en estudiantes universitarios. Revista de la educación superior, 43(169), 89-105. doi: 10.1016/j.resu.2015.01.003

Tornimbeni, S., Pérez, E., \& Olaz, F. (2008). Introducción a la psicometría. Buenos Aires: Paidós.

Vandenberg, R. J. (2002). Toward a further understanding of an improvement in measurement invariance methods and procedures. Organizational Research Methods, 5(2), 139-158. doi: $10.1177 / 1094428102005002001$
Vizcarro, C. (1994). Evaluación de las habilidades sociales. En: R., Fernández-Ballesteros. Evaluación conductual hoy: un enfoque para el cambio en psicología clínica y de la salud (pp. 347-384). Madrid: Ed. Pirámide.

$\mathrm{Yu}, \mathrm{C}$. Y. (2002). Evaluating cutoff criteria of model fit indices for latent variable models with binary and continuous outcomes. (Tesis doctoral). University of California, Los Angeles, USA. Extraída de http://www.statmodel.com/download/Yudisse rtation.pdf 